\title{
DC readout experiment in Enhanced LIGO
}

\author{
Tobin T Fricke,,${ }^{1, *}$ Nicolás D Smith-Lefebvre, ${ }^{2}$ Richard Abbott, ${ }^{3}$ \\ Rana Adhikari, ${ }^{3}$ Katherine L Dooley ${ }_{,}^{4}$ Matthew Evans, ${ }^{2}$ Peter \\ Fritschel, ${ }^{2}$ Valery V Frolov, ${ }^{5}$ Keita Kawabe, ${ }^{6}$ Jeffrey S Kissel, ${ }^{2}$ \\ Bram J J Slagmolen, ${ }^{7}$ and Sam J Waldman ${ }^{2}$ \\ ${ }^{1}$ Department of Physics and Astronomy, Louisiana State Univ., Baton Rouge, LA \\ ${ }^{2}$ LIGO Laboratory, Massachusetts Institute of Technology, Cambridge, MA \\ ${ }^{3}$ LIGO Laboratory, California Institute of Technology, MS 100-36, Pasadena, CA \\ ${ }^{4}$ Department of Physics, University of Florida, Gainesville, FL \\ ${ }^{5}$ LIGO Livingston Observatory, PO Box 940, Livingston, LA \\ ${ }^{6}$ LIGO Hanford Observatory, PO Box 159, Richland, WA \\ ${ }^{7}$ Australian National University, Canberra, ACT 0200, Australia \\ E-mail: tobin.fricke@aei.mpg.de
}

\begin{abstract}
The two $4 \mathrm{~km}$ long gravitational wave detectors operated by the Laser Interferometer Gravitational-wave Observatory (LIGO) were modified in 2008 to read out the gravitational wave channel using the DC readout form of homodyne detection and to include an optical filter cavity at the output of the detector. As part of the upgrade to Enhanced LIGO, these modifications replaced the radio-frequency (RF) heterodyne system used previously. We describe the motivations for and the implementation of DC readout and the output mode cleaner in Enhanced LIGO. We present characterizations of the system, including measurements and models of the couplings of the noises from the laser source to the gravitational wave readout channel. We show that noise couplings using DC readout are improved over those for $\mathrm{RF}$ readout, and we find that the achieved shot-noise-limited sensitivity is consistent with modeled results.

PACS numbers: 04.80.Nn, 07.60.Ly, 95.55.Ym
\end{abstract}

To be published in Class. Quantum Grav. 


\section{Introduction}

The Laser Interferometer Gravitational-wave Observatory[1] (LIGO) operates gravitational wave detectors at two sites in the United States (at Hanford, Washington and Livingston, Louisiana) as part of a global network of observatories with the goal of making a first direct detection of gravitational waves (GWs) and beginning an era of gravitational wave astronomy.

The LIGO facilities were designed to accommodate an initial detector design using the technology available at the time followed by a major upgrade known as Advanced LIGO[2]. The initial detectors reached design sensitivity in 2005 and subsequently completed a two year observational run (S5). Rather than continue to run in this configuration until the beginning of the Advanced LIGO upgrades in 2010, it was decided to upgrade the detectors opportunistically to an intermediate configuration known as Enhanced LIGO[3, 4, 5]. Enhanced LIGO culminated with LIGO's sixth science run (S6), which took place between July 2009 and October 2010 using this new configuration.

This paper describes two of the changes made in Enhanced LIGO: implementation of an optical filter cavity (the output mode cleaner) at the interferometer's output port, and implementation of a new technique to extract the gravitational wave signal from the interferometer, called $D C$ readout.

\section{Experimental arrangement}

Each LIGO detector is a Michelson interferometer with $4 \mathrm{~km}$ long arms consisting of Fabry-Perot resonant cavities (see figure 1). To increase the amount of laser power at the beamsplitter and in the interferometer arms, a power recycling mirror is used to direct the light reflected from the Fabry-Perot Michelson back into the interferometer. The combination of the power recycling mirror and the Michelson forms the power recycling cavity (PRC). The positions of the mirrors are combined into several degrees of freedom: the difference of the arm cavity lengths (DARM), the common (mean) arm cavity length (CARM), the short Michelson (the difference of the distances from the beamsplitter to two arm cavity input mirrors; $\mathrm{MICH}$ ), and the power recycling cavity (formed by the mean distance to the two arm input mirrors; PRC).

A suitably polarized gravitational wave incident on the detector produces differential phase modulation in the two arms, which is resonantly enhanced by the Fabry-Perot cavities. The resulting phase modulation sidebands interfere constructively at the beamsplitter and exit at the output port, while transmission of the carrier to the output port is suppressed by operating the Michelson near its dark fringe.

To sense the signal sidebands induced by a GW with a photodiode, it is necessary to introduce an additional local oscillator (LO) field to produce power variations that are detected by the photodiode. The initial generation of laser interferometer gravitational wave detectors used a heterodyne detection scheme[6] inspired by the Pound-DreverHall technique[7]. In this scheme, the local oscillator field consists of two strong radio- 


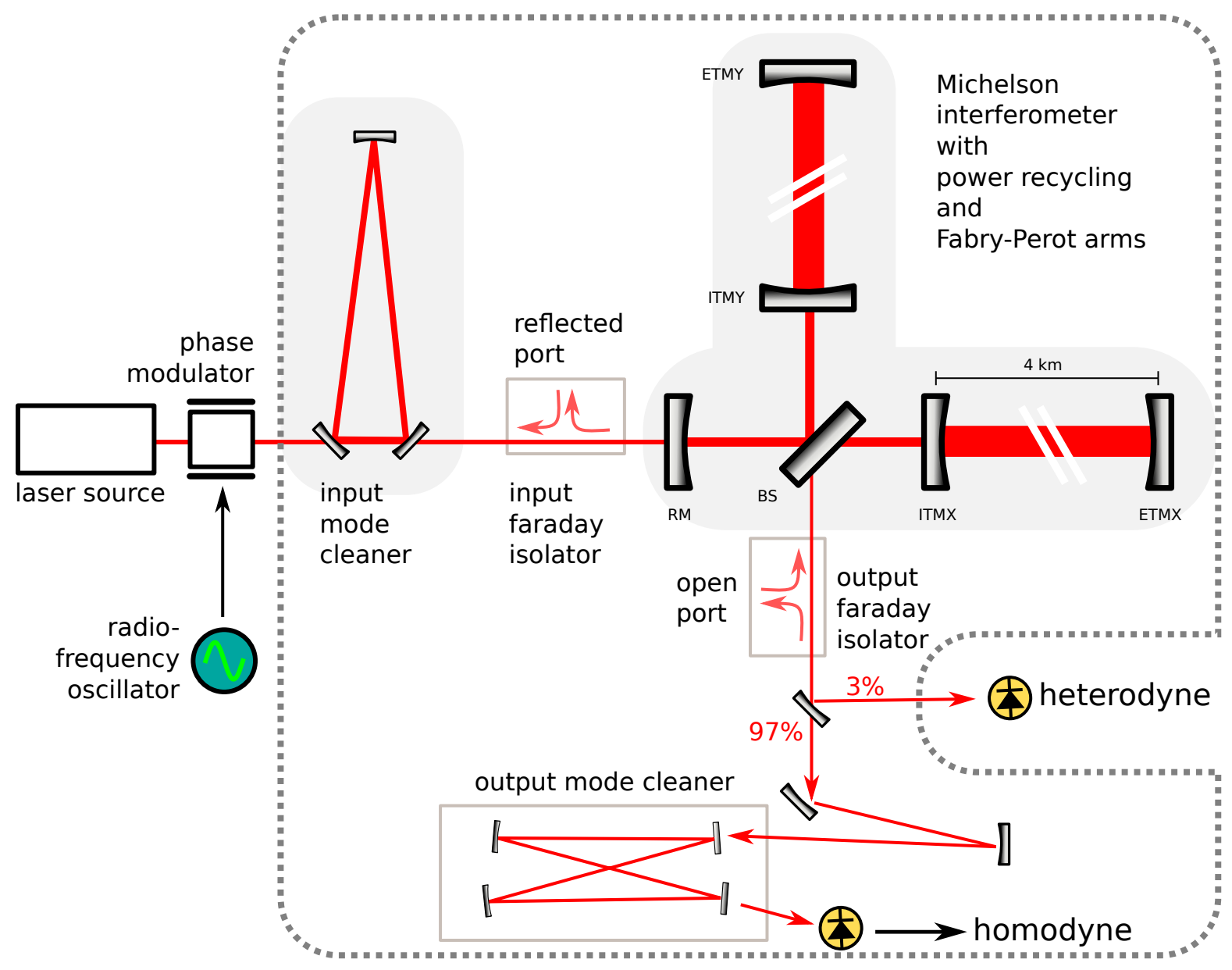

Figure 1: Schematic diagram of the main interferometer layout (not to scale). The dotted line represents the vacuum envelope.

frequency $(\mathrm{RF})$ sidebands separated from the laser carrier by tens of $\mathrm{MHz}(25 \mathrm{MHz}$ in initial/Enhanced LIGO). The interference between these local oscillator sidebands and the GW-induced sidebands produces a power modulation at the RF frequency whose amplitude is modulated by the GW amplitude. The GW signal is recovered by electronically demodulating the photodiode signal at the RF frequency. This scheme is known as $R F$ readout. Typically (and in LIGO), the RF sidebands are created by phase modulating the laser light before it enters the interferometer. To allow these sidebands to reach the output port while simultaneously suppressing transmission of the carrier, the short Michelson is built with a macroscopic difference in its arm lengths known as the Schnupp asymmetry (in initial/Enhanced LIGO, $355 \mathrm{~mm}$ ). By detecting light from other interferometer ports, RF readout can also be used to sense other degrees of freedom, such as the short Michelson, the length of the power recycling cavity, and the mean length of the two arm cavities 8 .

An alternative method of recovering the GW signal is to provide a local oscillator at the carrier frequency, an arrangement known as homodyne detection. The interference between the carrier-mode $\mathrm{LO}$ and the $\mathrm{GW}$-induced sidebands produces a power variation 
on the photodiode that directly reproduces the GW signal. DC readout is a special form of homodyne detection where the carrier-mode local oscillator is produced by introducing a static offset in either the short Michelson or in the difference between the arm cavity lengths, either of which will produce carrier light at the output port (but with different consequences for noise couplings). DC readout has two significant advantages over balanced homodyne detection[9], in which the local oscillator is provided by an independent path and combined with the signal beam via a beamsplitter: no additional optical path is needed to deliver the local oscillator to the output port, and the local oscillator that is generated benefits from being filtered by the combined action of the power recycling and arm cavities.

There are also a few potential drawbacks to DC readout as compared to balanced homodyne detection. In the DC readout configuration, the phase of the local oscillator relative to the signal field cannot be tuned directly; it is determined by the relative amplitudes of the intentional offset and the contrast defect. The static carrier field at the interferometer output port results in an unintended coupling of beam motion to the wavefront sensor at the output port, used by the interferometer's automatic alignment system[10]. Finally, detuning the arm cavities from resonance introduces an optical spring effect, which may lead to excessive coupling of laser intensity noise via radiation pressure effects in some configurations[11].

The optical fields in the interferometer are intended to exist in only the fundamental Gaussian spatial mode. A critically coupled resonant cavity, the input mode cleaner, is used to attenuate any higher order spatial modes produced by the laser before the field is incident on the interferometer [12, 13]. Despite having an essentially pure input beam, imperfections in the interferometer optics lead to the production of higher order spatial modes in the interferometer; this effect is particularly egregious for the RF sidebands in the power recycling cavity [14]. As a result, the output beam (depicted in figure 2 is no longer in the pure Gaussian mode. These higher order spatial modes are detrimental as they generally produce no useful signal, but contribute additional photon shot noise, increase the power that needs to be detected, and exacerbate noise couplings. To mitigate these effects, an output mode cleaner (OMC) was installed at the output port. This critically-coupled optical filter cavity attenuates higher order spatial modes before the beam is detected by a pair of photodiodes. In DC readout, the OMC is also used to remove the RF sidebands, which are still needed in the interferometer to sense other degrees of freedom, but would only be detrimental to the DC readout signal.

Both DC readout and an OMC have been implemented previously, but this was the first use of the combination on a km-scale GW detector with Fabry-Perot arms. Before implementing these technologies on the main LIGO interferometers, they were prototyped at the 40 meter prototype interferometer at Caltech [15, 16]. To see the full benefit of DC readout required an interferometer with long arms in order to achieve filtering of the laser carrier at the frequencies of interest; Enhanced LIGO provided validation of the low-noise performance of DC readout.

DC readout was simultaneously implemented and is currently used at the GEO 600 


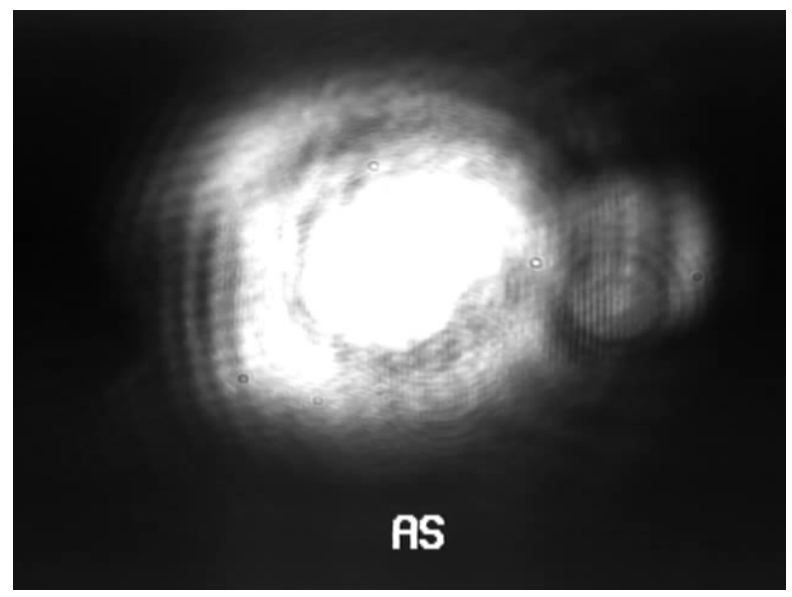

Figure 2: Image of the beam spot at the L1 output port taken using a CCD camera. This image is saturated in the central portion but emphasizes the spurious higher order modes surrounding the fundamental Gaussian, including contributions from both the carrier and the $25 \mathrm{MHz}$ sidebands.

detector [17, 18, 19, 20] near Hannover, Germany. The current configuration of the Virgo detector near Pisa, Italy incorporates an output mode cleaner but uses RF heterodyne readout[21]. Both Advanced Virgo[22] and Advanced LIGO[23] will use DC readout to sense the primary GW signal.

\section{Motivation}

The RF readout technique was used successfully in initial LIGO to achieve the instruments' design sensitivity. A number of reasons, outlined below, motivated the switch to DC readout with an OMC:

\subsection{Improved noise couplings}

The combination of the power recycling cavity and the arm cavities acts as a single resonant cavity with an effective linewidth of approximately $1 \mathrm{~Hz}$ (the so-called coupled cavity pole) around the laser carrier. The RF sidebands, which are not resonant in the arms, experience no such filtering in the band of interest 24. DC readout exploits this filtering by using the carrier light that has circulated in the interferometer as the local oscillator. The result is that coupling of noises on the input beam to the gravitational wave readout can be greatly reduced.

\subsection{Spatial overlap}

Because the signal beam (resonant in the arm cavities) and the RF sideband beam (resonant in the power recycling cavity) are resonant in different cavities, they will emerge from the interferometer in different spatial modes if these cavities are not 
perfectly matched. This imperfect spatial overlap was a significant problem during initial LIGO. For instance, a measurement in 2003 found only half the expected optical gain 25] (the ratio of photodiode signal produced to modulation of the differential arm length). This was alleviated in part through the use of a thermal compensation system (TCS), which projected light from a $\mathrm{CO}_{2}$ laser onto the test mass optics to adjust their effective radii of curvature, compensating for thermal lensing[26]. However, spurious fields still caused problems by producing a large signal in the uncontrolled quadrature of the heterodyne readout (AS_I); left uncontrolled, this signal would saturate the photodiode electronics. This was partially mitigated by an electronic servo which cancelled this signal in the photodiode head.

Both DC readout and an OMC mitigate problems with the spatial overlap of the LO and signal beams. In DC readout, the local oscillator and the signal beams resonate in the same cavities, so spatial overlap comes naturally. With either DC or RF readout, an OMC, matched to the spatial mode of the signal beam, can be used to select the signal beam and the part of the LO with good spatial overlap.

\subsection{Excess power}

To cope with the excess power due to higher-order modes at the interferometer output port, initial LIGO split the light at the detection port onto four detection photodiodes. Increasing the interferometer input power (to improve the sensitivity in the shot-noiselimited frequency band) would require a commensurate increase in the number of photodiodes at the output port and their associated electronics. This is of particular concern for Advanced LIGO, which will utilize a 200 Watt laser source in place of Enhanced LIGO's 35 Watt laser. An output mode cleaner attenuates the higher-order modes, reducing the power that needs to be detected.

\subsection{Homodyne SNR advantage}

Homodyne detection confers a fundamental improvement in signal-to-noise ratio

compared to RF readout at shot-noise-limited frequencies by a factor $\sqrt{3 / 2}$ (for the same power circulating in the interferometer). The extra noise in heterodyne detection is a result of cyclostationary shot noise 27].

\subsection{Squeezed vacuum injection}

Squeezed vacuum injection is an attractive means to decrease the photon quantum noise in future interferometers by manipulating the quantum state of the vacuum field that enters the interferometer through the output port. Squeezed vacuum injection is more feasible in conjunction with homodyne detection than with $\mathrm{RF}$ readout, since it requires squeezing in only the audio band rather than at both audio and RF frequencies [28, 29]. Shot noise reduction via squeezed vacuum injection has been demonstrated at GEO [30] and an effort is currently underway to implement it at LIGO Hanford[31]. 


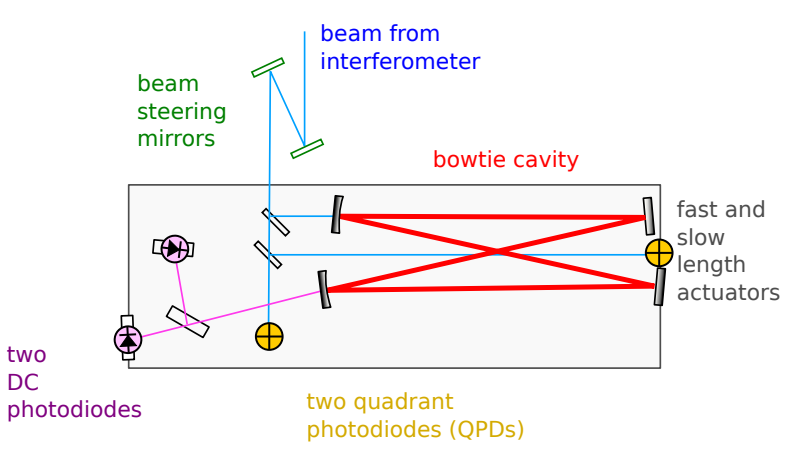

(a) Schematic of OMC bench

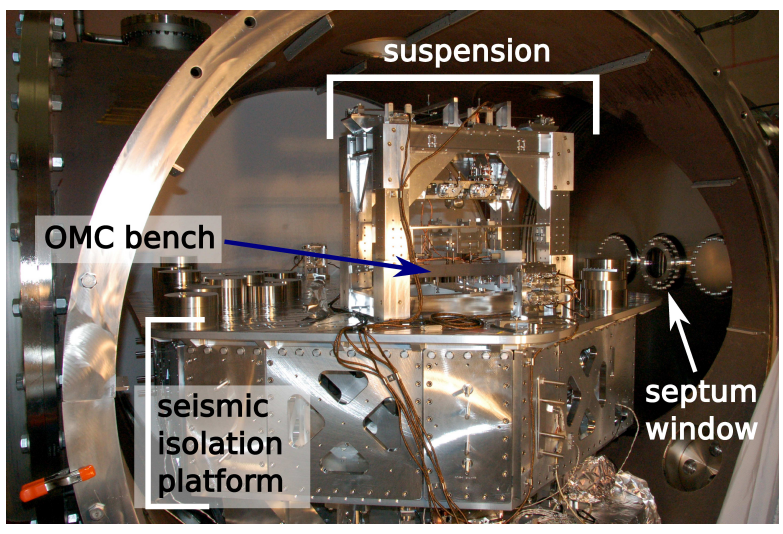

(b) Photograph of installed OMC and suspension

Figure 3: (a) Diagram illustrating the design of the monolithic OMC bench; (b) Photograph of the installed output mode cleaner, suspension, and seismic isolation platform. The OMC is located in a dedicated vacuum chamber, separated from the main vacuum enclosure by a septum window, allowing rapid venting cycles during commissioning.

\section{Implementation of DC readout}

The Enhanced LIGO output mode cleaner was installed in a dedicated vacuum chamber (photograph in figure 3b) at the interferometer's output port. Of the power arriving at the output port, $97 \%$ is directed to this new DC readout path while the remaining $3 \%$ is directed to the existing RF readout system. The control system used to maintain resonance uses the $\mathrm{RF}$ readout when initially bringing the system from an uncontrolled to a controlled ('locked') state, just as in initial LIGO[32]. Once the interferometer is held on resonance by the control system, we introduce an offset in the DARM degree of freedom, which produces carrier light at the output port. (The possibility of using a MICH offset to produce the local oscillator was not investigated for Enhanced LIGO, as modeling showed that a pure DARM offset would be effective.) The output mode cleaner is brought into resonance with this carrier light and then sensing of the DARM degree of freedom is transferred from the $\mathrm{RF}$ readout to the photodiodes sensing the light transmitted through the OMC.

To produce a sufficiently strong and stable local oscillator, the DARM offset must significantly exceed the RMS residual arm motion $(\sim 0.1$ picometers, depicted in figure (4), produce enough power at the output port such that the contrast defect becomes negligible, and provide a signal whose shot noise exceeds the electronics noise of the readout. Smaller DARM offsets are preferred, as a larger DARM offset results in diminished power recycling gain due to the power exiting the dark port, and because larger DARM offsets generally increase noise couplings. An optimum can thus be found.

In the homodyne system, a $\sim 30-100 \mathrm{~mW}$ local oscillator at the laser carrier frequency is instead introduced by offsetting the differential arm length (DARM) very 


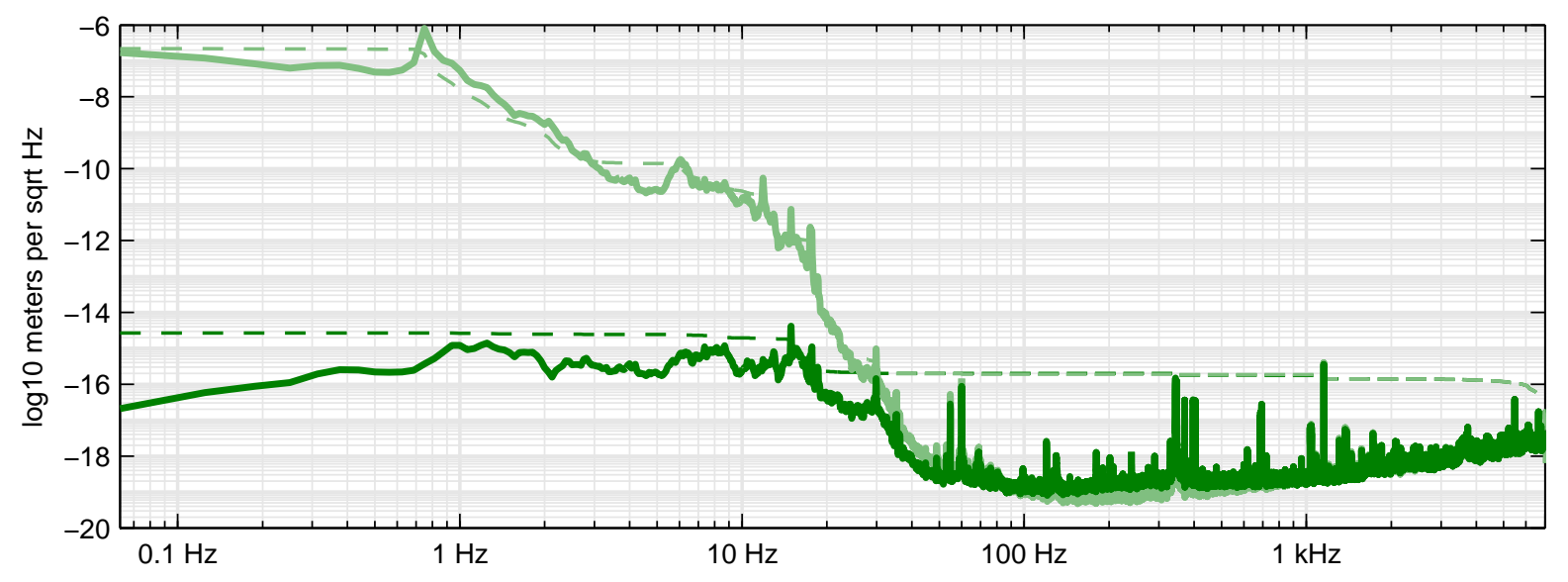

Figure 4: Calibrated DARM displacement (upper traces) and residual motion (lower traces), shown as amplitude spectral density (- - , in meters per $\sqrt{\mathrm{Hz}}$ ) integrated rootmean-square displacement (- - -, in meters). The RMS DARM displacement above $0.07 \mathrm{~Hz}$ is reduced by approximately eight orders of magnitude by the control system in order to remain sufficiently near the operating point. The residual motion sets a lower limit for the DARM offset in DC readout to avoid fringe-wrapping.

slightly ( $\sim 10$ picometers, equivalent to 60 microradians or $0.4 \mathrm{~Hz}$ detuning of each arm cavity, or 0.01 radians at the beamsplitter) from the dark fringe. The RF sidebands are no longer used for length sensing at the output port, but they still serve to provide sensing of some angular degrees of freedom.

\section{Mode Cleaner Design and Construction}

A four-mirror bow-tie arrangement (depicted in figure 3a) was chosen for the mode cleaner design. This non-colinear design prevents direct reflection of rejected light back into the interferometer. A design with an even number of mirrors was preferred as it has a sparser density of higher-order-mode resonances. Selecting the angle of incidence of the beam on the cavity mirrors is a compromise between avoiding small-angle scattering (by using a high angle of incidence) and avoiding the introduction of too much astigmatism.

A higher cavity finesse results in better filtering ability, but also magnifies the effect of any intra-cavity losses in the OMC. The cavity design maximizes the finesse subject to the constraint that transmission of the carrier power should exceed $99 \%$, assuming roundtrip cavity power losses of 100 ppm.

The cavity length was chosen to provide adequate attenuation of the RF sidebands, and its geometry ( $g$-factor) was chosen to sufficiently attenuate higher-order modes. The optimal $g$-factor depends on the specific details of the frequency and spatial spectrum of modes at the output port. These depend strongly on the details of the interferometer optics, alignment, and thermal state. To deal with these unknown factors, we designed the OMC using a model in which the power in each higher order mode was proportional 


\begin{tabular}{llllll}
\hline parameter & symbol & design & H1 & L1 & units \\
\hline perimeter & & 1.042 & 1.077 & 1.016 & $\mathrm{~m}$ \\
beam waist & $w$ & 477 & 496 & 463 & $\mu \mathrm{m}$ \\
finesse & $\mathcal{F}_{\text {omc }}$ & 400 & 360 & 360 & \\
free spectral range & $\nu_{0}$ & 287.7 & 278.3 & 295.2 & $\mathrm{MHz}$ \\
cavity pole & & 360 & 390 & 410 & $\mathrm{kHz}$ \\
$g$-factor & $g$ & 0.739 & 0.725 & 0.722 & \\
higher order mode spacing & & 69.4 & 67.2 & 71.8 & $\mathrm{MHz}$ \\
\hline
\end{tabular}

Table 1: Designed and measured properties of the Hanford and Livingston output mode cleaners.

to $1 / n^{2}$, where $n$ is the sum of the horizontal and vertical TEM mode indicies, and where the RF sidebands had their nominal power. The designed and as-built properties of the output mode cleaner cavities are given in Table 1 .

One disadvantage of the chosen design is that the 4-th order mode is nearly degenerate with the fundamental mode. We did experience problems with accidental degeneracy in one of the mode cleaners, which was addressed by changing the operating temperature of the thermal actuator (which had a small coupling to the effective radius of curvature of the mirror). The next version of the output mode cleaner will be designed with a slightly different g-factor to avoid this problem.

The cavity was constructed by rigidly mounting the cavity optics to a baseplate, similar to the LISA optical bench design [33. The baseplate is a slab of Corning ULE glass $450 \mathrm{~mm} \times 150 \mathrm{~mm} \times 39 \mathrm{~mm}$; components were bonded using Optocast 3553LV-UTFHM UV-cure epoxy. Two of the cavity mirrors are outfitted with position actuators: a fast, short-range $(\lesssim 0.1 \mu \mathrm{m}) \mathrm{PZT}$, and a slow, long-range $(\approx 20 \mu \mathrm{m})$ thermal actuator consisting of a 1 inch segment of aluminum tube warmed by a resistive heater.

To isolate the mode cleaner from environmental disturbances, the optical bench was hung as the second stage in an actively-damped double-pendulum suspension system, which was in turn mounted on an active isolation system[34, Chapter 5]. The active isolator, and the $\mathrm{OMC}$ and its suspension system, were all located in vacuum.

The photodiodes were also mounted on the OMC baseplate, and read out by in-vacuum preamplifiers. The output from the mode cleaner was split via a 50/50 beamsplitter and directed to two Perkin Elmer 3mm diameter InGaAs photodiodes (part number C30665GH), with measured quantum efficiency $>0.95$ at $1064 \mathrm{~nm}$. The photocurrent was converted to voltage across $100 \Omega$ transimpedance. Subtraction of the signals from the two photodiodes produces a diagnostic "nullstream" containing the anticorrelated component of the PD signals, while the sum of the photodiode signals contains the desired DARM sensing. The rigid mounting of the PDs to the OMC baseplate reduces the possibility of beam motion coupling to photocurrent through photodiode nonuniformities, and the in-vacuum preamplifiers reduce the effect of electronic or triboelectric noises. 
The mode cleaner was controlled and the DC readout signals were acquired using a prototype of the Advanced LIGO real-time digital signal processing system, operating at 32768 samples per second. The LIGO Realtime Code Generator 35] allowed fast prototyping and implementation of complex servos.

The mode cleaner cavity length was locked by modulating its length at $\sim 10 \mathrm{kHz}$ using the PZT actuator and synchronously demodulating the transmitted light signal at the same frequency. Fast corrections were applied to the PZT and then gradually offloaded onto the thermal actuator.

\section{Alignment system}

The beam from the interferometer output port is directed to the OMC by two suspended, steerable mirrors (depicted in figure 3a), prototypes of the Advanced LIGO steering mirrors described in [36]. These mirrors allow continuous actuation on the alignment of the beam from the interferometer to the OMC.

The same spurious higher order modes that motivate the use of an output mode cleaner also make it challenging to sense the best $\mathrm{OMC}$ alignment. A servo system that maximizes the intensity of the transmitted light is not optimal, since the presence of higher order modes will cause it to displace the input beam to convert some of the higher order modes into the cavity mode. Instead, a servo system was implemented to maximize the transmission of the arm cavity mode (containing the GW signal) through the OMC. This servo operates by driving one of the arm cavity end mirrors at high frequency $(9 \mathrm{kHz})$ while simultaneously modulating the two OMC beam steering mirrors at low frequency (1-4 Hz) in both pitch and yaw. The high-frequency modulation is accomplished by exciting the drumhead mechanical resonance of the cavity end mirror. The steering mirror modulation produces approximately 3 microns and 5 microradians of beam motion at the OMC cavity waist. An error signal is developed by measuring the power in the $9 \mathrm{kHz}$ line and demodulating this signal at the steering mirror modulation frequencies. An alternate alignment scheme, which maximizes the signal to noise ratio rather than the signal alone, has been proposed in [37].

\section{Results}

\subsection{Sensitivity}

The DC readout is dominated either by quantum shot noise or by signal (interferometer displacement noise) at all frequencies except sometimes near a few mechanical resonances causing beam motion (jitter), which couples linearly or bilinearly to the readout.

The shot-noise-limited noise floor depends both on the optical gain and the power at the AS port, making this a good measure of performance of the system. Both the presence of excess light at the detection port or sub-optimal optical gain will degrade the shot-noise-limited sensitivity. The expected shot noise amplitude spectral density, 

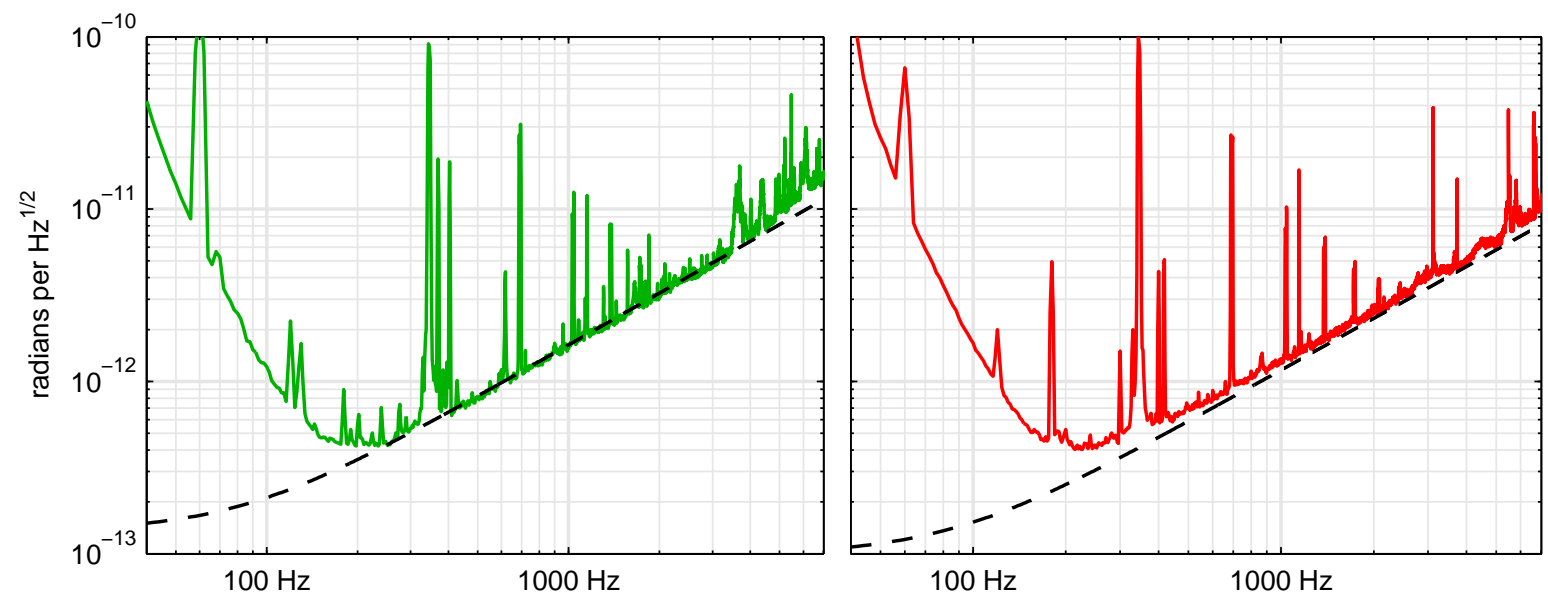

Figure 5: Achieved sensitivity (noise floor) amplitude spectral density for the Livingston (left) and Hanford (right) detectors ( - ), with expected shot noise limit (- - - -). The Livingston spectrum is from 2010-08-11 06:34 UTC and the Hanford spectrum is from 2010-07-04 08:52 UTC. The estimated uncertainty in the modeled curve is approximately $\pm 15 \%$.

expressed as a displacement $x_{\text {shot }}$ and an optical phase $\phi_{\text {shot }}$, is

$$
\begin{aligned}
& x_{\text {shot }}(f)=\frac{1}{4} \sqrt{\frac{\lambda h c}{2 \epsilon P_{I N}}} \frac{1}{g_{c r} \mathcal{F}_{\text {arm }}}\left|1+i \frac{4 \mathcal{F}_{\text {arm }} L}{c} f\right| \\
& \phi_{\text {shot }}(f)=\left(\frac{2 \pi}{\lambda}\right) x_{\text {shot }}(f)
\end{aligned}
$$

where $h$ is Planck's constant, $c$ is the speed of light, $P_{I N}$ is the power delivered by the laser source, $g_{c r}{ }^{2}$ is the power recycling gain, $\mathcal{F}_{\text {arm }}$ is the finesse of the arm cavities, $\epsilon$ is the input and output efficiency of the interferometer, and $\lambda$ is the laser wavelength. The parameters and efficiency used in the model are given in table 2 .

The achieved sensitivity is compared to the expected shot noise limit in figure 5 ; we find that the observed shot noise limit is consistent with the model. The agreement indicates that there is no significant excess shot noise due to higher-order modes, and the optical gain is as predicted; the benefits of homodyne detection with an OMC are achieved. However, sub-optimal transmission through the OMC due to imperfect mode-matching of the interferometer spatial mode into the OMC and/or increased OMC intra-cavity losses was one of the largest inefficiencies in the Hanford detector, equivalent to a $30 \%$ power loss and commensurate $15 \%$ increase in shot noise, partially negating the effect of Hanford's higher input power (see table 2).

\subsection{Noise couplings}

Noise couplings from the laser and RF oscillator are modified substantially by DC readout [15, 16, 38]. Other noise couplings (of displacement noises, in particular) generally remain the same. These couplings were measured at both Hanford and 


\begin{tabular}{llll}
\hline parameter & symbol & $\mathrm{H} 1$ & $\mathrm{~L} 1$ \\
\hline input power & $P_{I N}$ & $20.27 \mathrm{~W}$ & $11.65 \mathrm{~W}$ \\
arm cavity pole & $f_{c}$ & $83.7 \mathrm{~Hz}$ & $85.6 \mathrm{~Hz}$ \\
finesse & $\mathcal{F}_{\text {arm }}$ & 224 & 219 \\
power recycling gain & $g_{c r}{ }^{2}$ & 59 & 41 \\
\hline carrier fraction after phase modulation & $J_{0}(\Gamma)^{2}$ & 0.94 & 0.95 \\
input optics & & 0.82 & 0.75 \\
interferometer mode-matching & & 0.92 & 0.92 \\
output faraday isolator transmission & & 0.94 & 0.98 \\
DC readout pickoff fraction & & 0.953 & 0.972 \\
OMC mode-matching & & 0.70 & 0.95 \\
OMC transmission and PD quantum efficiency & & 0.95 & 0.95 \\
\hline net power efficiency & $\epsilon$ & 0.42 & 0.56 \\
\hline
\end{tabular}

Table 2: Interferometer parameters used in the shot noise model.

Livingston and compared to models.

To model the laser and oscillator noise transfer functions, we used the plane-wave frequency-domain interferometer simulation tool Optickle[39]. The simulation includes realistic parameters such as contrast defect and arm cavity finesse imbalance, and also includes the cross-couplings due to the servo control systems. However, the model does not include effects due to mode-matching or higher-order spatial modes.

DC readout also introduces at least two new noise sources: OMC path length fluctuations and beam pointing noise (jitter).

7.2.1. OMC path length noise Near resonance, the transmission of the $\mathrm{OMC}$ is a quadratic function of the intra-cavity optical path length. A change in OMC transmission is indistinguishable from a DARM perturbation; thus OMC path length noise can appear in the readout signal. Because the coupling is quadratic the contribution to the readout depends on the particular spectrum of path length noise. In practice, the dominant contribution tends to be via bilinear upconversion of lowfrequency motion. The root-mean-square deviation from resonance was found to be $\sim 75 \times 10^{-15} \mathrm{~m}$.

By adding band-limited random noise to the OMC PZT actuator and observing the effect in the transmitted light, and by comparing this coupling to the typical spectrum of the OMC path length error signal, we we estimated that the OMC path length noise contribution to DARM was further than $10 \times$ below the noise floor.

7.2.2. Beam jitter Beam jitter is perhaps the most important new noise source introduced in the DC readout system. The OMC converts motion of the incident beam into amplitude modulation, which pollutes the readout. In the ideal case, this would be a quadratic effect, but the presence of spurious higher order modes introduces both 
linear and bilinear contributions. We have several means of controlling the beam jitter contribution to the readout. In practice, we utilize all of them:

- Remove mechanical resonances and increase isolation. Several prominent beamjitter peaks in the readout spectrum were removed by replacing a fixed steering mirror with a suspended one; and by adding additional vertical isolation to the steering mirrors.

- Cancel the motion. Motion at the $60 \mathrm{~Hz}$ power-line frequency is introduced via magnetic coupling to the magnets used to actuate on the suspended steering mirrors. We implemented a feed-forward correction using signals derived from a magnetometer located just outside the vacuum chamber.

- Place steering optics where their coupling to beam jitter is small. Beam propagation geometry can magnify the effects of optic motion to beam motion. For a given amount of optic jitter, the coupling to readout noise can be reduced by proper design of the mode-matching telescope. This constraint is at odds with the desire for maximum controllability of the input beam pointing. In practice the requirement is that sufficient actuation range be provided, and that the optics be well separated in Gouy phase to create a non-degenerate control matrix.

- Reshape the beam. Linear beam jitter coupling arises due to the presence of spurious carrier light in the Hermite-Gauss TEM01 mode incident on the mode cleaner; displacement of the beam couples this mode into the fundamental Gaussian TEM00 mode of the mode cleaner cavity. By introducing offsets into the interferometer's global angular sensing and control (ASC) system[10, this spurious TEM01 mode can be minimized, reducing the linear sensitivity to beam motion. During Enhanced LIGO this was found to be a highly effective technique, but it was not automated.

An example of the observed beam jitter coupling is shown in figure 6. A prominent spectral line at $\sim 130 \mathrm{~Hz}$ appears in quadrant photodiode (QPD) signal, indicating relative motion in yaw between the incident beam and the OMC. This line also appears in the signal of the light transmitted through the OMC, indicating some linear coupling of beam jitter to transmission. The transmitted spectrum also contains sidebands around the $130 \mathrm{~Hz}$ line at separations of $\pm 0.875 \mathrm{~Hz}$ and $\pm 1.6 \mathrm{~Hz}$ which arise due to bilinear coupling.

7.2.3. Oscillator noises Although DC readout does not depend directly on the RF oscillator, the RF sidebands are still present in the interferometer (in particular at the output port, before the $\mathrm{OMC}$ ) and are used to sense other degrees of freedom. Noise on the RF sidebands can couple to DC readout both directly (by off-resonance transmission through the $\mathrm{OMC}$ ) and indirectly (via cross-couplings from other loops).

Oscillator amplitude modulation (AM) creates a time-varying modulation depth in the phase modulator used to produce the RF sidebands. In the frequency domain, this appears as anti-correlated AM on the carrier and the RF sidebands at the input to 

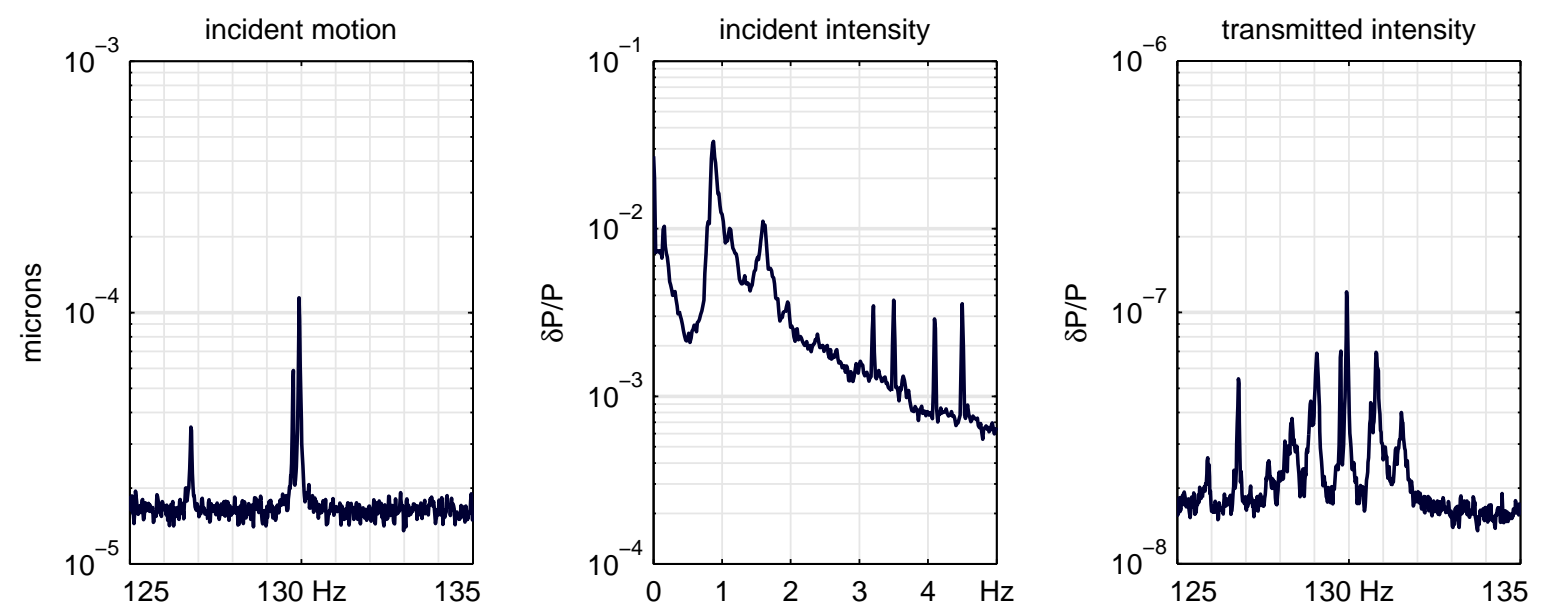

Figure 6: Example of linear and bilinear beam jitter coupling in the L1 OMC. Left: the light incident on the $\mathrm{OMC}$ shows motion at $\sim 130 \mathrm{~Hz}$. Center: the incident light contains intensity modulations at $\sim 0.87$ and $\sim 1.6 \mathrm{~Hz}$. Right: the light transmitted through the OMC contains intensity modulations at $130 \mathrm{~Hz}, 130 \pm 0.87 \mathrm{~Hz}$, and $130 \pm 1.6 \mathrm{~Hz}$. (The sources of the incident beam motion and intensity fluctuations at these frequencies have not been identified.)

the interferometer. These sidebands around the carrier and around the RF sidebands propagate differently through the interferometer and through the OMC: the sidebands around the carrier are filtered by the coupled cavity pole while those around the RF sidebands are not; conversely, the sidebands around the RF sidebands are strongly attenuated by the $\mathrm{OMC}$ while those around the carrier are transmitted. Varying carrier intensity also leads to a displacement noise via radiation pressure acting on the differentially detuned arm cavities. Oscillator phase noise, on the other hand, creates phase modulation sidebands around the RF sidebands, but does not produce any modulation of the carrier.

To measure the couplings of oscillator noises, we temporarily reverted to using a general purpose (IFR 2023A) RF function generator instead of the crystal oscillator as the interferometer's $25 \mathrm{MHz}$ oscillator. This function generator was configured to impress amplitude and phase modulation during swept-since measurements of the linear transfer functions from amplitude/phase modulation to the DC readout signal.

The results are depicted in figures 7 and 8 . We find that the DC readout sensitivity to RF oscillator amplitude and phase noises are reduced by a factor of 10-100 as compared to RF readout. The oscillator amplitude modulation (AM) coupling agrees well with the model. (We do not attempt to model the oscillator phase noise coupling, since it is strongly influenced by effects not modeled by Optickle.)

7.2.4. Laser noises Noise on the laser source appears on both the carrier and the RF sidebands. As in the discussion of oscillator noises, modulation of the carrier is attenuated by the coupled cavity pole while noise around the RF sidebands is attenuated 


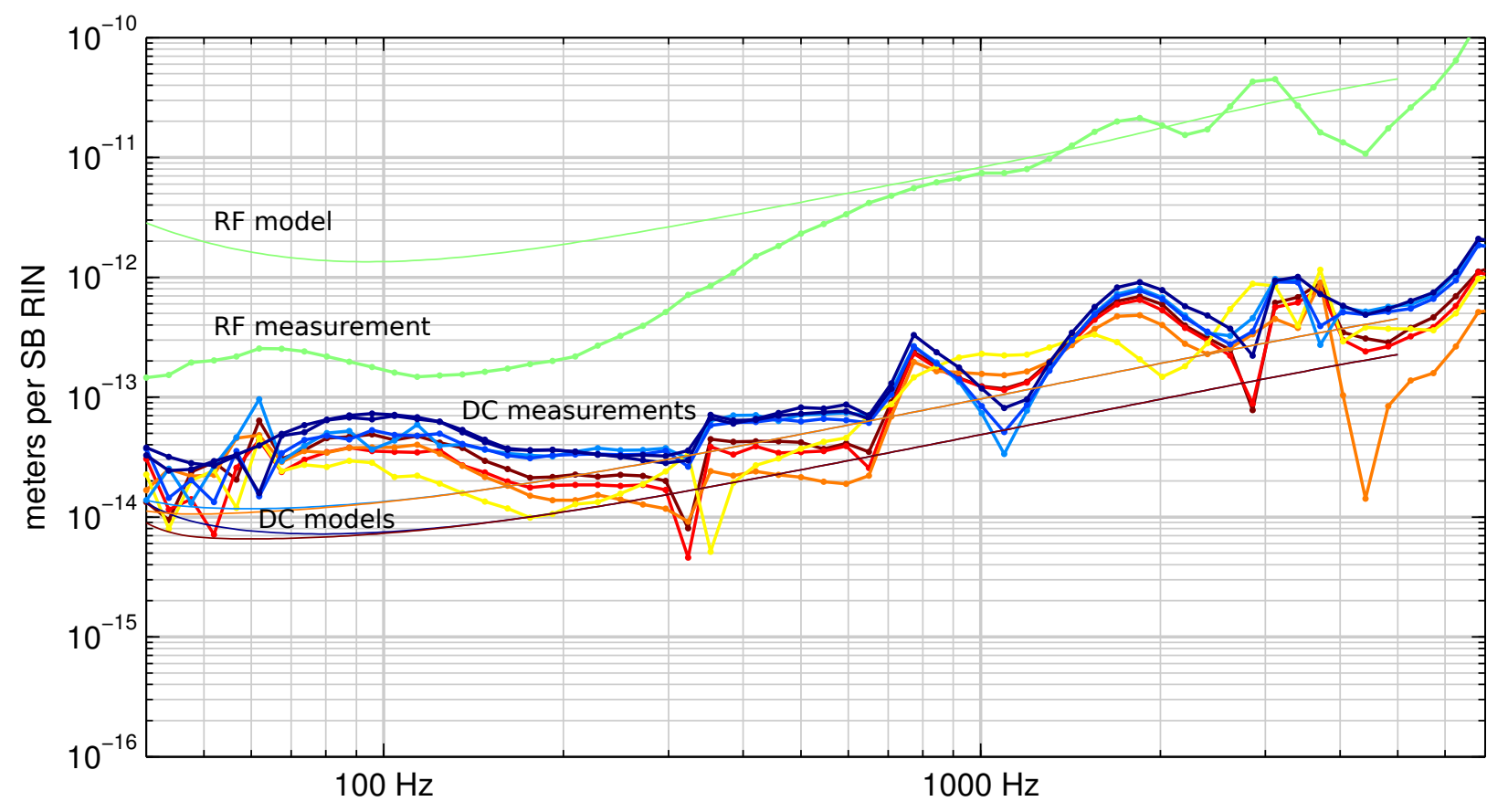

(a) Oscillator amplitude noise coupling (L1)

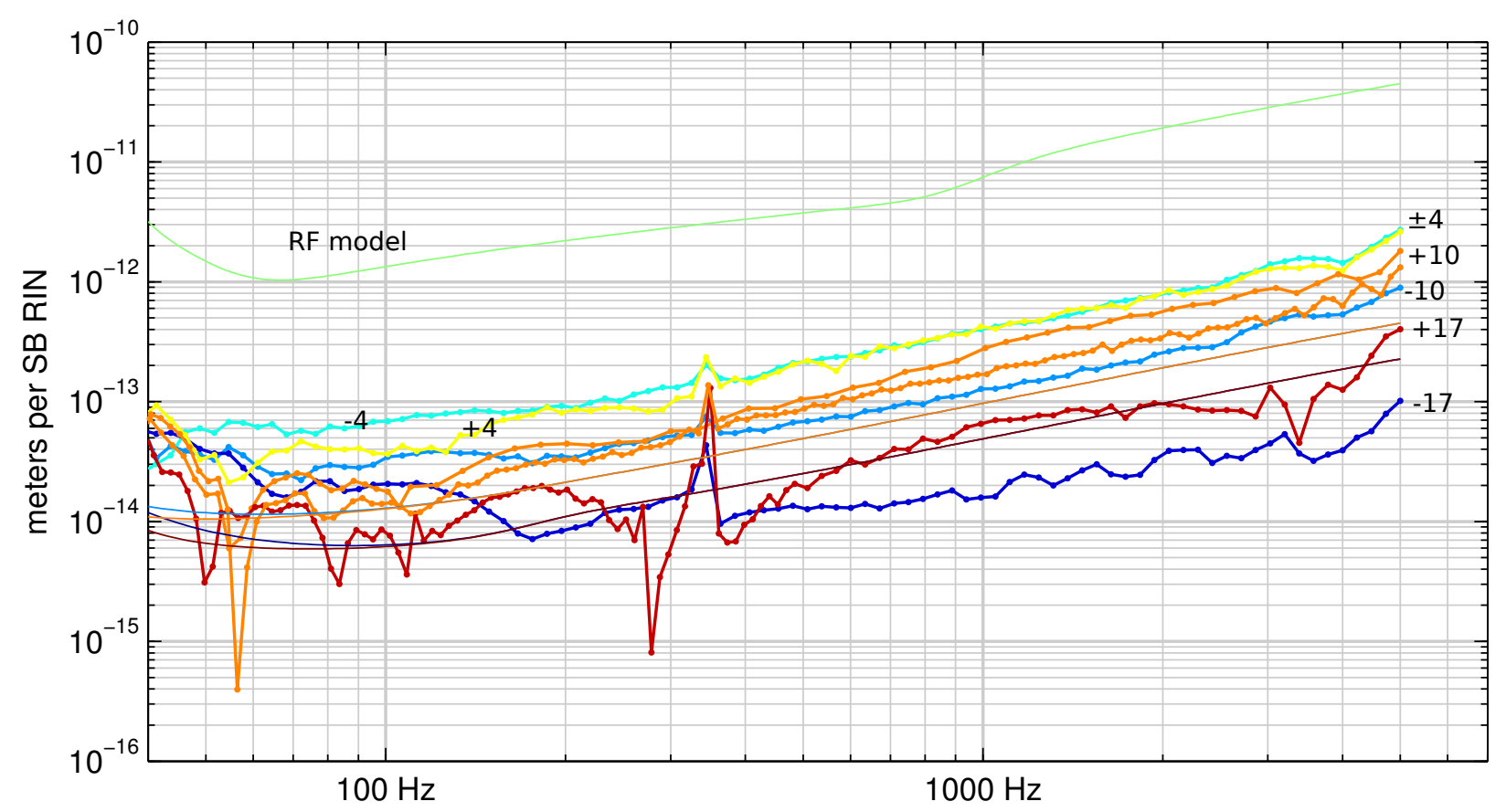

(b) Oscillator amplitude noise coupling (H1)

Figure 7: Oscillator amplitude noise couplings to the gravitational wave readout channel. Solid lines are the results of a frequency-domain, plane-wave model; dotted lines are linear transfer function measurements. Measurements were taken with DARM offsets between -20 and $+20 \mathrm{pm}$, and with $\mathrm{RF}$ readout (L1 only). Color represents the DARM offset, with warm colors for positive offsets and cool colors for negative offsets. Measurements and models for $\mathrm{RF}$ readout are in green. The vertical axis is equivalent DARM meters per relative intensity noise $(\delta P / P)$ of the RF sideband. 


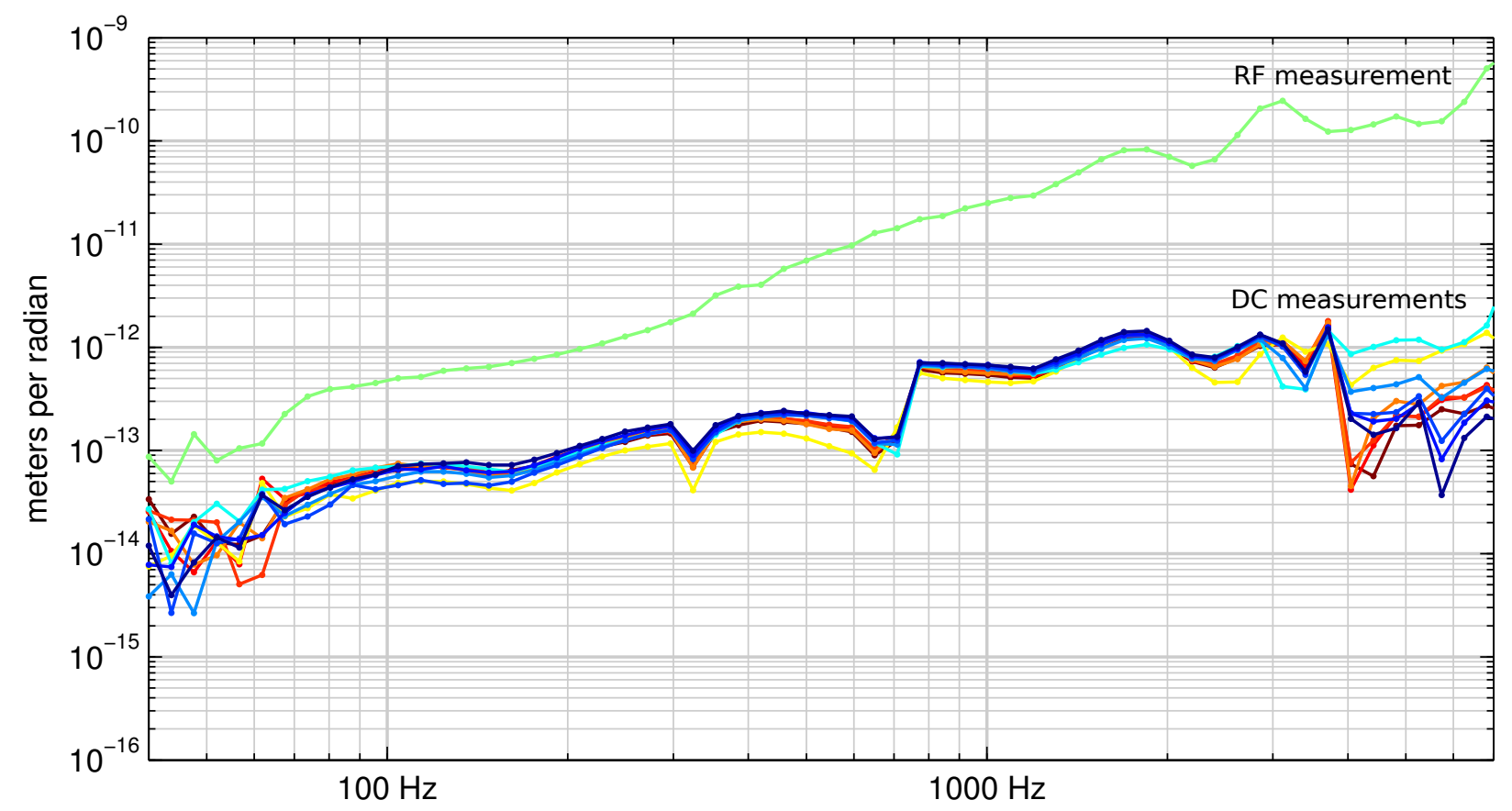

(a) Oscillator phase noise coupling (L1)

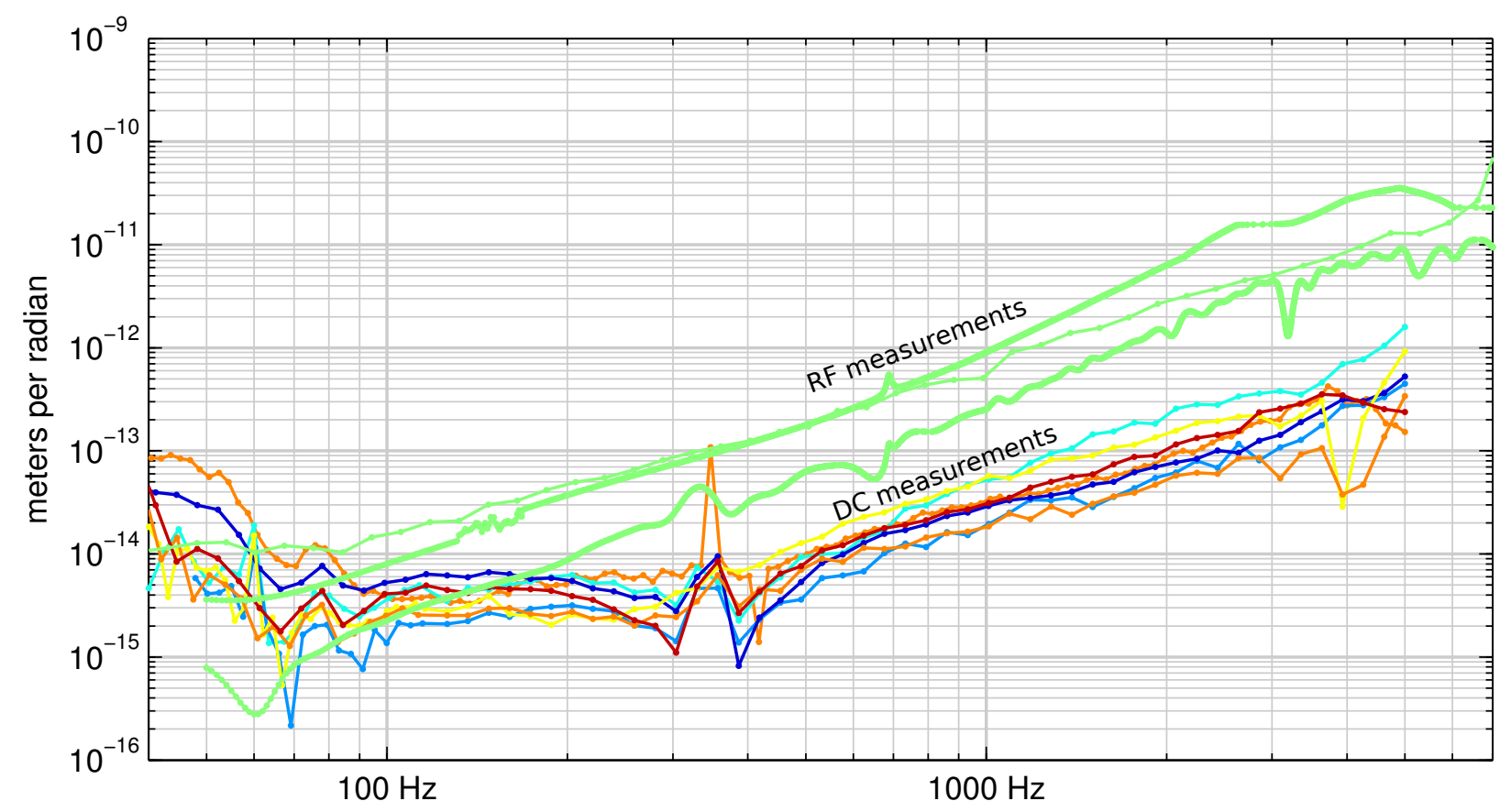

(b) Oscillator phase noise coupling (H1)

Figure 8: Oscillator phase noise couplings to the gravitational wave readout channel, calibrated in equivalent displacement (meters). All traces are linear transfer function measurements. Measurements were taken with DARM offsets between -20 and +20 pm, and with RF readout. Color represents the DARM offset, with warm colors for positive offsets and cool colors for negative offsets. Measurements and models for RF readout are in green. 


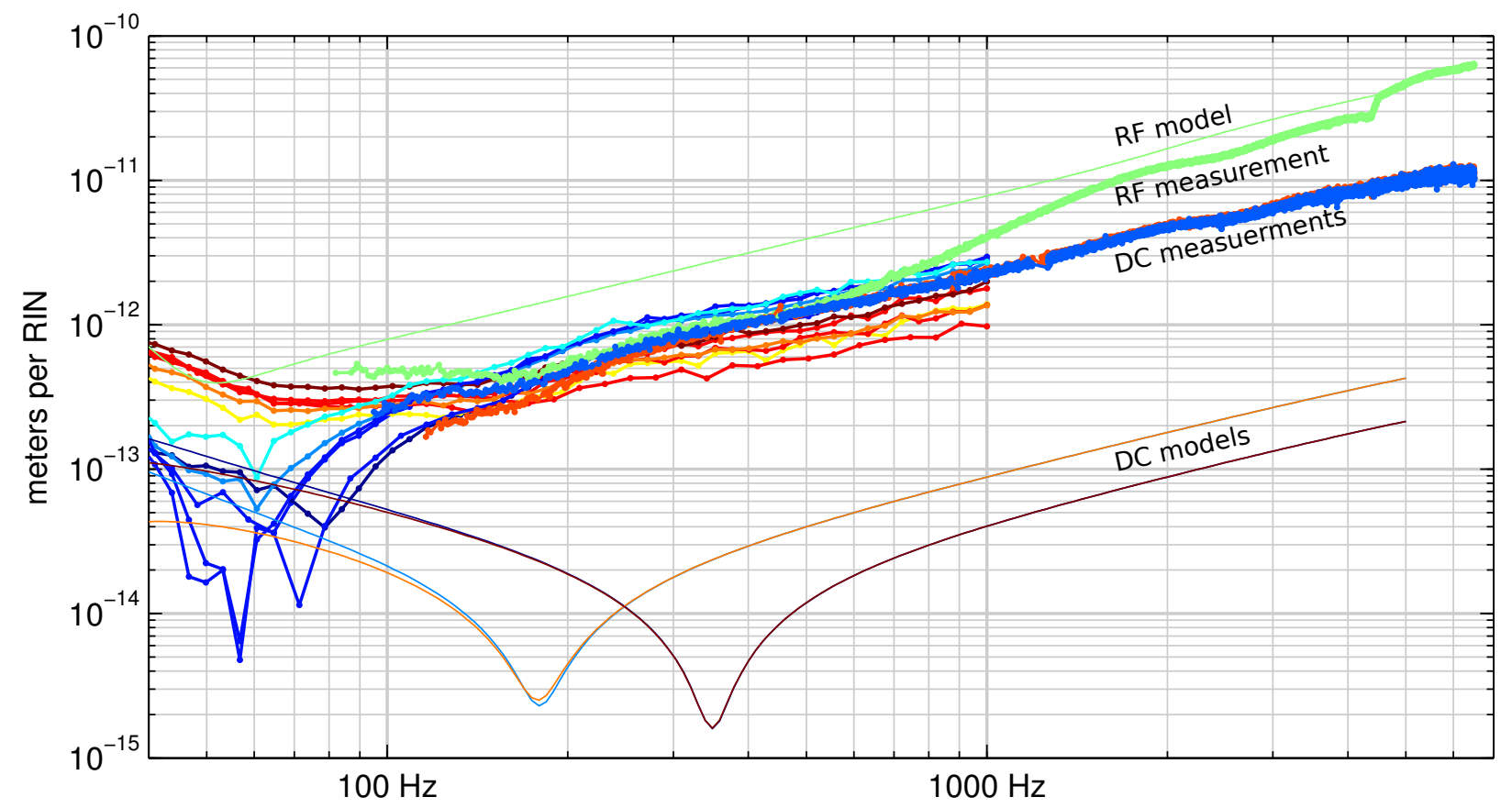

(a) Laser amplitude noise coupling (L1)

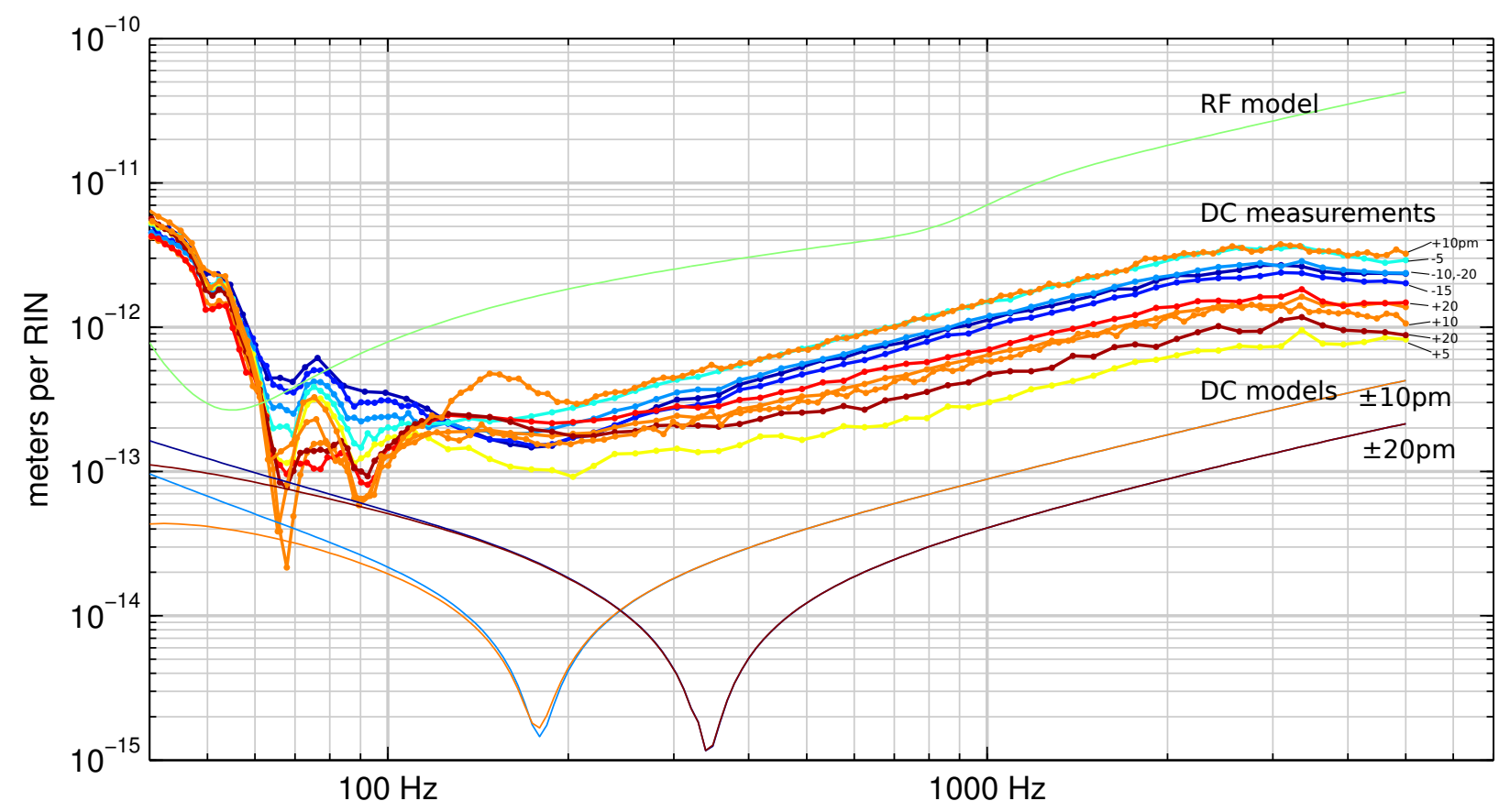

(b) Laser amplitude noise coupling (H1)

Figure 9: Laser amplitude noise couplings to the gravitational wave readout channel. Solid lines are the results of a frequency-domain, plane-wave model; dotted lines are linear transfer function measurements. Measurements were taken at DARM offsets between -20 to $+20 \mathrm{pm}$ and with $\mathrm{RF}$ readout (L1 only). Color represents the DARM offset, with warm colors for positive offsets and cool colors for negative offsets. Measurements and models for RF readout are in green. 


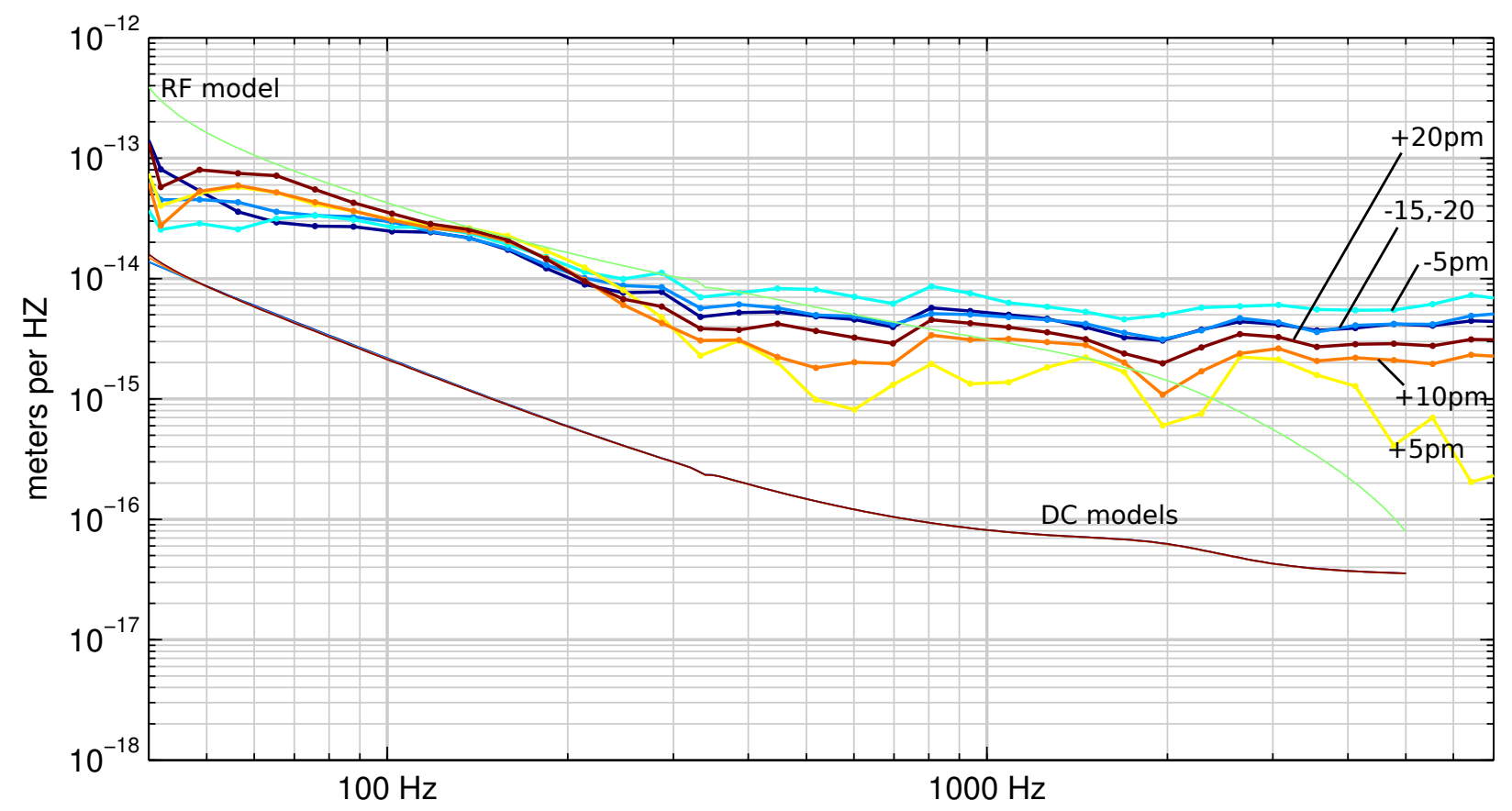

(a) Laser frequency noise coupling (L1)

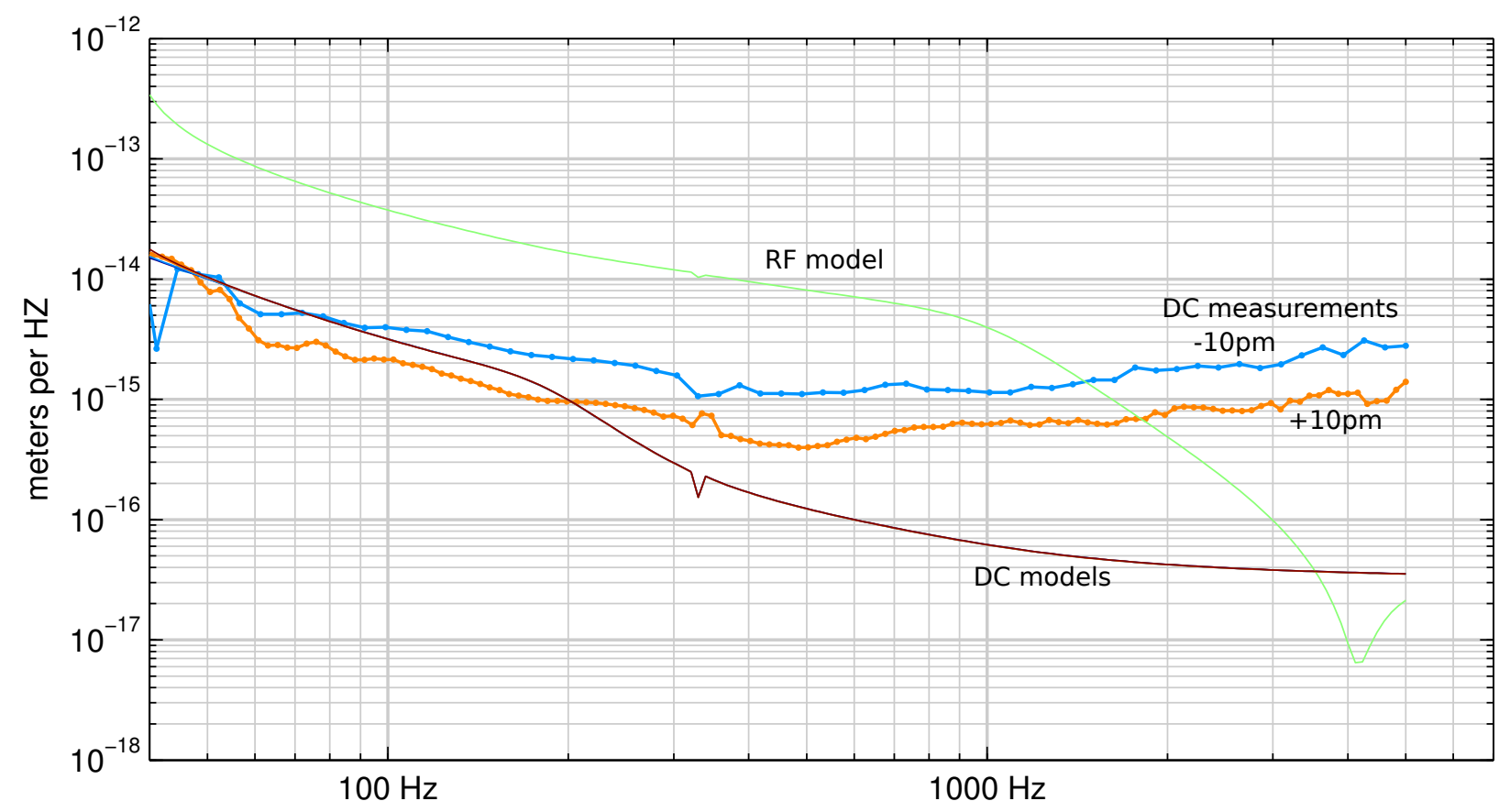

(b) Laser frequency noise coupling (H1)

Figure 10: Laser frequency noise couplings to the gravitational wave readout channel. Solid lines are the results of a frequency-domain, plane-wave model; dotted lines are linear transfer function measurements. Measurements were taken with DARM offsets of $\pm 20, \pm 10, \pm 5 \mathrm{pm}$ at L1 and $\pm 10 \mathrm{pm}$ at H1. Color represents the DARM offset, with warm colors for positive offsets and cool colors for negative offsets. 
by the OMC. Amplitude noise couples directly to DC readout, while frequency noise must be converted to AM by asymmetries in the interferometer to couple to the readout.

To measure the coupling of laser intensity noise, the intensity of the laser source was modulated by driving the error point of the laser intensity stabilization servo. The coupling of laser frequency noise to the readout was measured by driving the error point of the common mode servo (CARM), which nulls the mismatch between the laser frequency and the average of the two arm lengths by actuating on the laser frequency. We calibrate the CARM sensor by injecting a sinusoidal excitation into the drive to one of the end mirrors. By measuring the response in both CARM and DARM and compensating for the suppression due to the feedback control system, the DARM calibration is ported to CARM, which is then be converted from meters to $\mathrm{Hz}$ using the relation $\delta L / L=\delta \nu / \nu$ with $L=3995 \mathrm{~m}$ and $\nu=c / \lambda$.

The measured intensity noise and frequency noise transfer functions are depicted in figures 9 and 10, respectively. In both cases, the observed coupling is much greater than the model predicts. This coupling may be due in part to residual transmission of higher order spatial modes from imperfect alignment of the interferometer output beam to the OMC; carrier light in these higher order spatial modes does not resonate in the arms and thus is not attenuated by the coupled-cavity pole. Further evidence that this coupling is dominated by contributions due to higher-order modes is that it changed significantly between two measurements made a few months apart. The higher-order mode content is highly dependent on the thermal state of the interferometer and its coupling to DC readout depends on the alignment of the output beam into the OMC.

\section{Conclusion}

The Enhanced LIGO experiment has validated the DC readout scheme as a low-noise readout system scalable to high-power operation and alleviating some of the troubles experienced with RF readout. We have shown that the shot-noise-limited sensitivity achieved with DC readout is consistent with expectations, and presented measurements of laser and oscillator noise couplings to the gravitational wave channel. These couplings are generally much better with DC readout than with RF readout. We find that a simple plane wave model is not adequate to explain the laser noise couplings; future work should utilize a more complex model incorporating the effects of higher order spatial modes.

\section{Acknowledgments}

TF thanks the National Science Foundation for support under grant PHY-0905184 and Caltech for support via the LIGO Visitors Program. The authors would like to thank Koji Arai, Hartmut Grote, David Shoemaker, and Zach Korth for comments on this manuscript; and everyone who contributed to the Enhanced LIGO effort. LIGO was constructed by the California Institute of Technology and Massachusetts Institute of Technology with funding from the National Science Foundation and operates 
under cooperative agreement PHY-0757058. This article has LIGO Document Number P1000009.

\section{References}

[1] B. P. Abbott, et al. LIGO: the Laser Interferometer Gravitational-wave Observatory. Reports on Progress in Physics, 72(7):076901+, July 2009. doi:10.1088/0034-4885/72/7/076901.

[2] Alex Abramovici, William E. Althouse, Ronald W. P. Drever, Yekta Gursel, Seiji Kawamura, Frederick J. Raab, David Shoemaker, Lisa Sievers, Robert E. Spero, Kip S. Thorne, Rochus E. Vogt, Rainer Weiss, Stanley E. Whitcomb, and Michael E. Zucker. LIGO: The laser interferometer gravitational-wave observatory. Science, 256(5055):325-333, April 1992. doi: 10.1126/science.256.5055.325,

[3] Josh R. Smith for the LIGO Scientific Collaboration. The path to the enhanced and advanced LIGO gravitational-wave detectors. Classical and Quantum Gravity, 26(11):114013+, June 2009. arXiv:0902.0381, doi:10.1088/0264-9381/26/11/114013.

[4] Peter Fritschel, Rana Adhikari, and Rai Weiss. Enhancements to the LIGO S5 detectors. Technical Report T050252-00-I, LIGO Laboratory, November 2005. URL: http://www.ligo.caltech. edu/docs/T/T050252-00.pdf.

[5] Rana Adhikari, Peter Fritschel, and Sam Waldman. Enhanced LIGO. Technical Report T060156-01-I, LIGO Laboratory, July 2006. URL: http://www.ligo.caltech.edu/docs/T/ T060156-01.pdf.

[6] Peter Fritschel, Rolf Bork, Gabriela González, Nergis Mavalvala, Dale Ouimette, Haisheng Rong, Daniel Sigg, and Michael Zucker. Readout and control of a power-recycled interferometric gravitational-wave antenna. Appl. Opt., 40(28):4988-4998, October 2001. URL: http://www. ligo.caltech.edu/docs/P/P000008-A.pdf, arXiv:physics/0111047, doi:10.1364/A0.40. 004988

[7] R. W. P. Drever, J. L. Hall, F. V. Kowalski, J. Hough, G. M. Ford, A. J. Munley, and H. Ward. Laser phase and frequency stabilization using an optical resonator. Applied Physics B: Lasers and Optics, 31(2):97-105, June 1983. doi:10.1007/BF00702605.

[8] Martin W. Regehr. Signal Extraction and Control for an Interferometric Gravitational Wave Detector. PhD thesis, California Institute of Technology, August 1994. URL: http://www. ligo.caltech.edu/docs/P/P940002-00.pdf.

[9] Kirk McKenzie, Malcolm B. Gray, Ping K. Lam, and David E. McClelland. Technical limitations to homodyne detection at audio frequencies. Appl. Opt., 46(17):3389-3395, June 2007. doi: 10.1364/A0.46.003389,

[10] L. Barsotti, M. Evans, and P. Fritschel. Alignment sensing and control in advanced LIGO. Classical and Quantum Gravity, 27(8):084026+, April 2010. doi:10.1088/0264-9381/27/8/ 084026 .

[11] W. Chaibi and François Bondu. Optomechanical issues in the gravitational wave detector advanced VIRGO. Comptes rendus de l'Académie des Sciences, in press.

[12] A. Rüdiger, R. Schilling, L. Schnupp, W. Winkler, H. Billing, and K. Maischberger. A mode selector to suppress fluctuations in laser beam geometry. Optica Acta, 28:641-658, May 1981. URL: http://www.ligo.caltech.edu/docs/P/P800001-00.pdf, doi:10.1080/713820609.

[13] Katherine Dooley, Muzammil Arain, David Feldbaum, Valery Frolov, Matthew Heintze, Daniel Hoak, Em Khazanov, Antonio Lucianetti, Rodica Martin, Guido Müller, Oleg Palashov, Volker Quetschke, David H. Reitze, Rick Savage, David Tanner, Luke Williams, and Wan Wu. Characterization of thermal effects in the enhanced LIGO input optics. Review of Scientific Instruments, 2011.

[14] Andri M. Gretarsson, Erika D'Ambrosio, Valery Frolov, Brian O'Reilly, and Peter K. Fritschel. Effects of mode degeneracy in the LIGO livingston observatory recycling cavity. J. Opt. Soc. Am. B, 24(11):2821-2828, November 2007. arXiv:0708.3470, doi:10.1364/JOSAB.24.002821. 
[15] R. L. Ward, R. Adhikari, B. Abbott, R. Abbott, D. Barron, R. Bork, T. Fricke, V. Frolov, J. Heefner, A. Ivanov, O. Miyakawa, K. McKenzie, B. Slagmolen, M. Smith, R. Taylor, S. Vass, S. Waldman, and A. Weinstein. DC readout experiment at the Caltech $40 \mathrm{~m}$ prototype interferometer. Classical and Quantum Gravity, 25(11):114030+, 2008. doi:10. 1088/0264-9381/25/11/114030.

[16] Robert L. Ward. Length Sensing and Control of an Advanced Prototype Interferometric Gravitational Wave Detector. PhD thesis, California Institute of Technology, Pasadena, CA, February 2010. URL: http://thesis.library.caltech.edu/5836/

[17] S. Hild, H. Grote, J. Degallaix, S. Chelkowski, K. Danzmann, A. Freise, M. Hewitson, J. Hough, H. Luck, M. Prijatelj, K. A. Strain, J. R. Smith, and B. Willke. DC-readout of a signalrecycled gravitational wave detector. Classical and Quantum Gravity, 26(5):055012+, March 2009. doi:10.1088/0264-9381/26/5/055012.

[18] M. Prijatelj, H. Grote, J. Degallaix, M. Hewitson, S. Hild, C. Affeldt, A. Freise, J. Leong, H. Lück, K. A. Strain, H. Wittel, B. Willke, and K. Danzmann. Control and automatic alignment of the output mode cleaner of GEO 600. Journal of Physics: Conference Series, 228(1):012014+, May 2010. doi:10.1088/1742-6596/228/1/012014.

[19] J. Degallaix, H. Grote, M. Prijatelj, M. Hewitson, S. Hild, C. Affeldt, A. Freise, J. Leong, H. Lück, K. A. Strain, H. Wittel, B. Willke, and K. Danzmann. Commissioning of the tuned DC readout at GEO 600. Journal of Physics: Conference Series, 228(1):012013+, May 2010. doi:10.1088/1742-6596/228/1/012013.

[20] M. Prijatelj, J. Degallaix, H. Grote, J. Leong, C. Affeldt, S. Hild, H. Lück, J. Slutsky, H. Wittel, K. Strain, and K. Danzmann. The output mode cleaner of GEO 600. Classical and Quantum Gravity, 2012.

[21] F. Acernese, et al. The Virgo $3 \mathrm{~km}$ interferometer for gravitational wave detection. Journal of Optics A: Pure and Applied Optics, 10(6):064009+, June 2008. doi:10.1088/1464-4258/10/ 6/064009.

[22] The Virgo Collaboration. Advanced virgo baseline design. Technical Report VIR-0027A-09, Virgo Collaboration, August 2009. URL: https://tds. ego-gw.it/ql/?c=6589.

[23] Rich Abbott, Rana Adhikari, Stefan Ballmer, Lisa Barsotti, Matt Evans, Peter Fritschel, Valera Frolov, Guido Mueller, Bram Slagmolen, and Sam Waldman. Advanced LIGO interferometer sensing and control conceptual design. Technical Report T070247, LIGO Laboratory, April 2008. URL: https://dcc.ligo.org/cgi-bin/DocDB/ShowDocument?docid=5306

[24] J. B. Camp, H. Yamamoto, S. E. Whitcomb, and D. E. McClelland. Analysis of light noise sources in a recycled Michelson interferometer with Fabry-Perot arms. J. Opt. Soc. Am. A, 17(1):120128, January 2000. URL: http://www.ligo.caltech.edu/docs/P/P000004-00.pdf, doi: 10.1364/JOSAA.17.000120

[25] Peter Fritschel. Shot noise and optical gain of the H1 interferometer. Technical Report T030004-00-D, LIGO Laboratory, January 2003. URL: http://www.ligo.caltech.edu/docs/ T/T030004-00.pdf.

[26] Stefan Ballmer. LIGO interferometer operating at design sensitivity with application to gravitational radiometry. PhD thesis, Massachusetts Institute of Technology, June 2006. URL: http://dspace.mit.edu/handle/1721.1/36396.

[27] T. M. Niebauer, R. Schilling, K. Danzmann, A. Rüdiger, and W. Winkler. Nonstationary shot noise and its effect on the sensitivity of interferometers. Physical Review A, 43(9):5022-5029, May 1991. doi:10.1103/PhysRevA.43.5022.

[28] J. Gea-Banacloche and G. Leuchs. Squeezed states for interferometric gravitational-wave detectors. Journal of Modern Optics, 34(6):793-811, 1987. doi:10.1080/09500348714550751.

[29] Simon Chelkowski, Henning Vahlbruch, Karsten Danzmann, and Roman Schnabel. Coherent control of broadband vacuum squeezing. Physical Review A, 75(4):043814+, April 2007. URL: http://www.ligo.org/pdf_public/chelkowski01.pdf, doi:10.1103/PhysRevA.75.043814.

[30] J. Abadie, et al. A gravitational wave observatory operating beyond the quantum shot-noise limit. 
Nature Physics, advance online publication, September 2011. doi:10.1038/nphys2083.

[31] Daniel Sigg, Nergis Mavalvala, David McClelland, Ping K. Lam, Roman Schnabel, Henning Vahlbruch, and Stan Whitcomb. Proposal for a squeezed H1 interferometer. Technical Report T070265-D, LIGO Laboratory, October 2008. URL: http://www.ligo.caltech.edu/docs/T/ T070265-D/T070265-D.pdf.

[32] M. Evans, N. Mavalvala, P. Fritschel, R. Bork, B. Bhawal, R. Gustafson, W. Kells, M. Landry, D. Sigg, R. Weiss, S. Whitcomb, and H. Yamamoto. Lock acquisition of a gravitationalwave interferometer. Opt. Lett., 27(8):598-600, April 2002. URL: http://authors.library. caltech.edu/5527/1/EVAol02.pdf, doi:10.1364/OL.27.000598.

[33] L. d'Arcio, J. Bogenstahl, M. Dehne, C. Diekmann, E. D. Fitzsimons, R. Fleddermann, E. Granova, G. Heinzel, H. Hogenhuis, C. J. Killow, M. Perreur-Lloyd, J. Pijnenburg, D. I. Robertson, A. Shoda, A. Sohmer, A. Taylor, M. Tröbs, G. Wanner, H. Ward, and D. Weise. Optical bench development for LISA. In International Conference on Space Optics. European Space Agency, October 2010. URL: http://congrex.nl/icso/Papers/Session\% 2014b/FCXNL-10A02-2017738-1-weise_icso_paper.pdf

[34] Jeffrey S. Kissel. Calibrating and Improving the Sensitivity of the LIGO Detectors. PhD thesis, Louisiana State University, September 2010. URL: http://etd.lsu.edu/docs/available/ etd-09122010-101812/.

[35] Rolf Bork and Jay Heefner. ELIGO OMC front end controls design description. Technical Report T0900329, LIGO Laboratory, July 2009. URL: https://dcc.ligo.org/cgi-bin/ DocDB/ShowDocument?docid=3492.

[36] Bram J. J. Slagmolen, Adam J. Mullavey, John Miller, David McClelland, and Peter Fritschel. Tip-tilt mirror suspension: Beam steering for advanced laser interferometer gravitational wave observatory sensing and control signals. Review of Scientific Instruments, 82(12):125108+, December 2011. doi:10.1063/1.3669532.

[37] N. Smith-Lefebvre, S. Ballmer, M. Evans, S. Waldman, K. Kawabe, V. Frolov, and N. Mavalvala. Optimal alignment sensing of a readout mode cleaner cavity. Optics Letters, 36(22):4365+, November 2011. doi:10.1364/0L.36.004365.

[38] K. Somiya, Y. Chen, S. Kawamura, and N. Mio. Frequency noise and intensity noise of nextgeneration gravitational-wave detectors with RF/DC readout schemes. Physical Review D, 73(12):122005+, June 2006. doi:10.1103/PhysRevD.73.122005.

[39] Matthew Evans. Optickle. Technical Report T070260-00, LIGO Laboratory, October 2007. URL: http://www.ligo.caltech.edu/docs/T/T070260-00.pdf 